Meta

Journal des traducteurs

Translators' Journal

\title{
L'interprétation pour sourds au pénal en France
}

\section{Christiane Fournier}

Volume 42, numéro 3, septembre 1997

L’interprétation en langues des signes

URI : https://id.erudit.org/iderudit/002764ar

DOI : https://doi.org/10.7202/002764ar

Aller au sommaire du numéro

\section{Éditeur(s)}

Les Presses de l'Université de Montréal

ISSN

0026-0452 (imprimé)

1492-1421 (numérique)

Découvrir la revue

Citer cet article

Fournier, C. (1997). L'interprétation pour sourds au pénal en France. Meta, 42(3), 533-545. https://doi.org/10.7202/002764ar

\section{Résumé de l'article}

L'interprétation pour sourds est une grande inconnue, non seulement pour le grand public, mais également pour bien des magistrats. Cette inconnue repose sur une double méconnaissance : • Qu'est-ce que l'interprétation ? • Quelles sont les incidences de la surdité, pour celui qui en est frappé, au niveau de ses potentialités de communication avec autrui ? Ces méconnaissances peuvent engendrer involontairement des conséquences préjudiciables au justiciable sourd tant sur l'affaire jugée que sur son vécu psychologique en fonction du regard posé sur lui. Par incidence, ces méconnaissances entraînent des jugements très divers, voire faux, à l'égard de l'interprétation et de l'interprète. À travers mon article, je me propose d'expliciter notre activité interprétative en mettant en évidence les problèmes spécifiques liés à la surdité, en les insérant dans le contexte particulier du milieu judiciaire. 


\title{
L'INTERPRÉTATION POUR SOURDS AU PÉNAL EN FRANCE
}

\author{
CHRISTIANE FOURNIER \\ Université Paris III-Sorbonne Nouvelle (ESIT) et Centre National d'Études et \\ de Formation pour l'Enfance Inadaptée, Paris, France
}

\begin{abstract}
Résumé
L'interprétation pour sourds est une grande inconnue, non seulement pour le grand public, mais également pour bien des magistrats. Cette inconnue repose sur une double méconnaissance:
\end{abstract}

- Qu'est-ce que l'interprétation?

- Quelles sont les incidences de la surdité, pour celui qui en est frappé, au niveau de ses potentialités de communication avec autrui?

Ces méconnaissances peuvent engendrer involontairement des conséquences préjudiciables au justiciable sourd tant sur l'affaire jugée que sur son vécu psychologique en fonction du regard posé sur lui. Par incidence, ces méconnaissances entraînent des jugements très divers, voire faux, à l'égard de l'interprétation et de l'interprète.

À travers mon article, je me propose d'expliciter notre activité interprétative en mettant en évidence les problèmes spécifiques liés à la surdité, en les insérant dans le contexte particulier du milieu judiciaire.
Abstract
The public at large, and more specifically, judges are unaware of the intricacies of interpretation. Interpreting is misunderstood and so are the repercussions of deafness on a deaf person's capacity to communicate.
This misunderstanding is likely to seriously affect legal decisions as well as the mental state of the deaf person involved in the legal process.
It also has a bearing on interpretation in general and on the interpreter's performance in particular.
In this article, I shall explain the court interpreter's task and discuss related problems posed by deafness.

\section{LE POIDS DU PASSÉ}

L'interprétation pour les personnes sourdes auprès des tribunaux a pris naissance, en France, vers les années 1830, à la demande d'une minorité intellectuelle de sourds, en vue de faire respecter les droits de tout citoyen sourd comme ceux de tout citoyen d'un même pays.

Pour ne pas faillir aux droits de l'homme, les magistrats ont reconnu l'intérêt légitime de cette interprétation et l'ont consignée par l'article 345 du Code pénal.

Toutefois, au vu de l'histoire de la communauté sourde et du discrédit dont souffrait la langue des signes, il n'est pas surprenant que l'interprétation pour sourds se soit limitée pendant plus d'un siècle au secteur judiciaire - force est à la loi et nécessité oblige.

L'interprétation était considérée comme une assistance à de malheureux «sourdsmuets» par un transcodage de tout message parlé en un langage mimique, assuré par des parents ou des professeurs. Rien qui puisse s'apparenter aux prestigieuses interprétations des langues orales. 
Il a fallu une véritable révolution des mentalités de notre société à l'égard des personnes sourdes, un nouveau jugement sur la langue des signes et ses potentialités conceptuelles, pour permettre à l'interprétation en langue des signes de conquérir ses lettres de noblesse en devenant une véritable profession. Depuis environ deux décennies, en France, l'interprétation pour les personnes sourdes a gravi des échelons sur le chemin du professionnalisme.

Cette ascension résulte de la convergence de plusieurs facteurs dont la reconnaissance de la langue des signes a été le détonateur. La communauté sourde a exercé des pressions légitimes pour pouvoir bénéficier d'interprétations aussi bien à titre privé que lors de congrès, séminaires ou manifestations en tout genre. Ces requêtes ont créé une situation nouvelle; si les personnes sourdes en sont les artisans, les interprètes en sont, chaque jour, les acteurs.

Le professionnalisme s'est substitué à un «assistanat interprétatif» perpétué depuis des lustres, situation qu'ont connue la plupart des pays et que certains connaissent encore. Ce transfert a été réalisable grâce à la prise de conscience par les interprètes eux-mêmes de la nécessité d'une formation de haut niveau, d'une parfaite maîtrise des langues et d'un bagage cognitif sérieux tant en connaissances générales qu'en connaissance du monde de la surdité. Seules ces conditions réunies peuvent garantir la qualité de l'interprétation et la fiabilité du message retransmis.

Le nouveau regard de notre société sur les sourds, la renaissance de la langue des signes après un siècle d'ostracisme ont constitué un terrain favorable pour souligner la nécessité de l'interprétation entre personnes sourdes et entendantes. Par leurs compétences professionnelles et par la confiance qu'ils ont su créer, les interprètes ont contribué à élargir leur champ d'intervention dans les domaines les plus variés.

Notre activité s'assimile en de nombreux points à celle de nos confrères interprètes en langues vocales. Ce qui pourrait nous différencier reposerait sur les origines des demandes. Le secteur social, ce qui se comprend de par les problèmes posés par la surdité, nous sollicite plus que l'Unesco, l'OCDE, le Parlement européen, ou tout autre organisme international.

Toutefois, un domaine est commun à tous les interprètes, quelles que soient les langues de travail: l'interprétation au pénal.

Tout interprète au pénal met en œuvre une activité interprétative qui repose sur le principe fondamental de toute interprétation, à savoir «comprendre pour se faire comprendre» (Lederer 1973: 21), c'est-à-dire appréhender le sens du vouloir-dire exprimé par autrui et le rendre intelligible à celui qui ne partage pas la même langue. Mais la spécificité de l'interprétation au pénal est liée aux problèmes humains, à la rigidité du cadre judiciaire et aux obstacles linguistiques inhérents à des niveaux socio-culturels différents, déclivité culturelle entre émetteur et récepteur. Ces obstacles, qu'affrontent également les interprètes des langues orales, sont notre lot quotidien et ont un effet grossissant sur l'ensemble des problèmes de l'interprétation au pénal.

\section{DE LA FONCTION À LA PROFESSION D'INTERPRÈTE}

Si l'interprétation pour sourds a pris naissance dans les tribunaux et a servi de tremplin pour une extension professionnelle, elle supporte le poids du passé et connaît encore maintes difficultés pour se libérer des préjugés pesant sur la personne sourde et sur son mode de communication. Ces entraves insidieuses trouvent leur point d'ancrage dans l'article 345 du Code de procédure pénal:

Si l'accusé est sourd-muet et ne sait pas écrire, le président nomme d'office en qualité d'interprète la personne qui a le plus d'habitude à converser avec lui. Il en est de même à l'égard du témoin sourd-muet. Dans le cas où le sourd-muet sait écrire, le greffier rédige les 
questions ou observations qui lui sont faites; elles sont remises à l'accusé ou au témoin, qui donne par écrit ses réponses ou déclarations. Il est fait lecture du tout par le greffier.

Même si les magistrats ne se réfèrent plus aujourd'hui à cet article, il n'en demeure pas moins qu'il est toujours en vigueur malgré les demandes d'abrogation et la rédaction d'un nouvel article. L'énoncé actuel met en évidence la méconnaissance de ce qu'est l'interprétation et de ses implications, du rôle de l'interprète, et des incidences de la surdité sur le savoir-lire et le savoir-écrire.

Habitude de communiquer ne signifie pas aptitude à interpréter. Si telle était la définition de l'interprétation, toutes les libertés pourraient être prises par rapport au message initial. Ni fidélité ni impartialité ne pourraient être garanties et le message retransmis serait un compromis entre le transcodage, l'exégèse ou un résumé minimal.

L'interprétation est un acte de communication authentique assuré par un interprète qui tient un rôle de médiateur entre deux locuteurs ne partageant pas la même langue. Elle est la réexpression intelligible et fidèle d'un vouloir-dire. Mais fídélité ne renvoie pas à l'idée de miroir de la langue. Une activité interprétative n'est pas une activité mécanique, comme me le laissait entendre un jour un magistrat: «On ne vous demande pas de comprendre, on vous demande de traduire.» C'est une activité intellectuelle qui implique de comprendre le sens du message du locuteur pour le restituer dans son intégralité au destinataire indépendamment des signes linguistiques qui ont composé le message initial.

Peut-on différencier l'interprétation de conférence de l'interprétation auprès des tribunaux pénaux, puisqu'une interprétation est toujours une interprétation et que les qualités requises pour assurer un travail de qualité sont les mêmes dans un cas comme dans l'autre? Si les compétences des interprètes doivent être effectivement les mêmes dans les deux domaines d'interprétation, le rôle de l'interprète auprès des tribunaux, les conditions de travail, les obstacles linguistiques, la prise en compte de situations humaines souvent délicates, pénibles, sont des facteurs qui requièrent des aptitudes particulières et qui nécessitent des stratégies d'interprétation adaptées aux circonstances.

Une analyse détaillée permettra de mettre en évidence les spécificités qui caractérisent l'interprétation au pénal.

\section{L'INTERPRÉTATION EN L.S.F. AU PÉNAL : SES SPÉCIFICITÉS}

\subsection{LES PRÉLIMINAIRES AU DÉROULEMENT DU PROCÈS}

Les tribunaux représentent les lieux où sont jugés crimes et délits. Au-delà de l'acte, ils sont le théâtre de problèmes humains avec leur cortège de drames.

Déjà en franchissant la porte de la salle d'audience, bien des prévenus vivent angoisses, inquiétudes sur l'issue de leur procès, et éprouvent une gêne de voir leur vie privée étalée au grand jour. Par leur caractère austère et impressionnant, les lieux exercent une pression psychologique sur toute personne appelée à la barre, a fortiori sur les prévenus. Pour les prévenus non francophones, du fait qu'ils ne partagent pas la même langue, voire la même culture, la peur de ne pas comprendre ou de ne pas être compris augmente leur anxiété. Le prévenu sourd vit en plein désarroi, en quête du moindre indice pour se sécuriser: sa nationalité française masque la nécessité d'un interprète. Elle ne s'impose pas systématiquement à l'esprit des personnes non averties. D'où la hantise de celui-ci si aucun interprète n'est convoqué ou ne s'est manifesté dès le début de l'audience. De plus, lorsque son nom sera cité, comment le saura-t-il? Il ne peut l'entendre alors que le non-francophone entendant pourrait, lui, l'entendre. Aussi, il gardera les yeux rivés sur le visage de l'huissier pour pouvoir lire son nom sur les lèvres lorsque celui-ci sera prononcé. En cas d'impossibilité, selon la place qu'il occupe dans la salle d'audience, il tentera de repérer les indices qui pourraient l'informer sur son sort. 
Le rôle de l'interprète commence dès le moment où il a remis sa convocation à l'huissier. Sa première mission est d'entrer en contact avec le justiciable, dans la mesure du possible, sans arrière-pensée d'apitoiement sur son cas ou tout autre sentiment qui pourrait entacher la totale impartialité du message à retransmettre. Face à l'interprète, le prévenu éprouve un sentiment de soulagement qui se lit sur son visage. Ce premier contact est important, car il contribue à améliorer les conditions de communication et il offre l'occasion de tester le mode de langage utilisé par la personne sourde.

Ces préliminaires font partie du travail préparatoire de l'interprète en permettant, dès le premier échange, une meilleure concentration sur le contenu des messages sans se disperser sur des problèmes extralinguistiques qui peuvent pourtant surgir à tout moment au cours du procès.

Lorsque l'affaire est citée, avant que tout dialogue ne débute, l'interprète doit savoir bien se placer pour s'assurer tout à la fois une bonne perception auditive et une bonne visibilité du message: presque de face par rapport au prévenu et non côte à côte comme l'est l'interprète de langue vocale - et surtout ne pas tourner le dos à la lumière.

S'il n'a pu le faire en aparté, l'interprète doit demander l'autorisation au tribunal de s'assurer des potentialités communicationnelles du prévenu: des diversités existent selon les degrés de surdité et selon les méthodes éducatives. Le tribunal accepte toujours ce bref échange dans l'intérêt d'une bonne interprétation. De cet échange, l'interprète doit savoir s'il est en mesure d'assumer son rôle et de répondre à l'attente des deux interlocuteurs. En cas d'impossibilité ou de difficultés particulières, par respect pour le code déontologique, il doit en informer le tribunal qui avisera.

\subsection{LES CONDITIONS D'ÉNONCIATION}

Les conditions d'énonciation sont liées au contexte, à la personnalité du prévenu et, du fait de sa surdité, elles requièrent de sa part une vigilance de chaque instant, le regard favorisant la prise d'indice dans un processus communicationnel. La prise de parole par le prévenu est liée à la demande du président d'audience, qui lui est signifiée par l'interprète. Au cours des différentes phases d'audience de tout procès, les locuteurs changent: grâce à la voix, les personnes entendantes autochtones ou étrangères peuvent orienter leur regard vers celui qui parle. Que les propos soient tenus dans une langue connue ou inconnue, le message perçu est facilement attribuable à son auteur. La personne sourde n'a pas cette faculté d'orientation, d'où l'obligation pour l'interprète de toujours indiquer qui reprend la parole en situant le locuteur avant de restituer le message.

\subsection{LES CONNAISSANCES NÉCESSAIRES}

Pour comprendre un discours, un réquisitoire, un plaidoyer ou de courts énoncés d'un interrogatoire, l'interprète doit avoir des compétences linguistiques et extralinguistiques. Celles-ci englobent des connaissances juridiques et contextuelles. De ce fait, des informations concernant l'affaire jugée sont absolument nécessaires au préalable; or elles sont rarement communiquées, même sur demande de l'interprète, sous le couvert du secret de l'instruction. Les chefs d'accusation notifiés sur la feuille de convocation sont les seules informations connues, mince bagage cognitif pour pouvoir être opérationnel dès les premiers énoncés. Est-ce la crainte d'un manque de neutralité, ou l'inutilité de cette requête ? Difficile à dire. Combien de fois ai-je entendu: «L'affaire est simple, vous n'aurez aucun problème particulier, il vous suffit de traduire.»

Pourquoi un minimum d'informations est-il nécessaire? Les magistrats connaissent le dossier, le justiciable est le premier concerné dans l'affaire; il existe un savoir partagé, la communication pourrait passer, même avec des implicites. Mais si l'interprète est hors du savoir partagé, il risque, surtout pour les premières phrases, de tâtonner avant de bien 
comprendre le vouloir-dire du locuteur, et il ne pourra transmettre un message suffisamment intelligible, ce qui, indirectement, peut créer un climat de malaise ou de méfiance. D'où la nécessité d'informations préalables comme conditions requises à toute activité interprétative. Si l'interprète a prêté son concours lors des commissions rogatoires, sa tâche sera facilitée. Mais il n'en est pas toujours ainsi.

Après la vérification d'identité du prévenu, le président invite ce dernier à s'expliquer sur les faits ou sur les motifs qui l'ont poussé à commettre le délit pour lequel il a été amené à comparaître devant le tribunal. Dans bien des cas, sans aucune explication circonstanciée, nous sommes en pleine action sous forme d'images séquentielles. Traduction littérale de propos tenus lors de son procès par monsieur T.: «Ma femme crie mon bébé pleure... ma maison tout désordre ... moi colère ... ma femme mon enfant, moi protège.» Chaque unité de sens constitue une information mais sans lien explicite entre elles. Comment transmettre un message cohérent et authentique? L'absence d'information sur le contexte nous oblige à formuler des hypothèses en prenant en compte les chefs d'accusation. En l'occurrence, il s'agissait de coups et blessures sur la personne de gendarmes. On peut subodorer qu'une perquisition avait été ordonnée au domicile de cet homme. A-t-elle été effective? Pour quelles raisons? Était-il lui-même présent? En a-t-il constaté les effets une fois celle-ci terminée? Mais savait-il ce qu'était une perquisition et connaissait-il les lois qui la régissent? J'en doute fort. Toutefois, une certitude dans le flou de ces énoncés: la. colère et la peur de ce père de famille qui était apparemment affolé ; sentiment qu'il transmet par ces mots : «Moi protéger ma femme et mon enfant.» Fallait-il comprendre : «J'ai frappé pour protéger les miens»? Mais il ne le dit pas et, de plus, n'évoque nullement la présence des gendarmes. Bien des implicites peuvent étayer chaque unité de sens.

À ce stade de connaissances, nous devons susciter des questions pour éclairer le sens du vouloir-dire. J'expliciterai cette problématique dans les stratégies de l'interprétation, qui aident à mieux cadrer les lieux, savoir si des personnes sont impliquées et tisser des relations de cause à effet. La transmission d'énoncés très morcelés pourrait émaner d'une personne parlant sous le coup de la colère, de l'indignation, et les faits ne peuvent que corroborer cette impression. En fait, elle traduit surtout des formulations maladroites dues à une déclivité intellectuelle dont les magistrats ne soupçonnent pas toujours l'amplitude. C'est à l'interprète de faire la part de ce qui revient au message et de la part de ce qui revient à la psychologie sans empiéter sur ce domaine.

\subsection{LE NOTIONNEL ET L'ÉMOTIONNEL}

Le contenu d'un message ne se limite pas à l'aspect notionnel. Si l'on exclut certains discours techniques à caractère purement informatif, où l'émotion n'a guère sa place, l'aspect émotionnel s'intègre au sens du message. On ne peut dissocier les traits prosodiques des éléments linguistiques rationnels d'un message. C'est de cette fusion des composants du discours que l'interprète saisira toute la portée du vouloir-dire et saura, par le ton ou le geste, habillant ses mots ou ses signes, être le médiateur fidèle de la parole d'autrui. Danica Seleskovitch (1976: 66 et 73), en mettant en évidence l'aspect notionnel et émotionnel d'un discours, a fait apparaître le «rapport entre le conscient et le réflexe dans la communication»: «Seul le sens est constamment présent en conscience; la volonté de le communiquer agit comme une pulsion qui mobilise, dans la langue maternelle, tous les moyens d'expression qui contribueront à sa transmission: traits prosodiques, pointu de la voix, registre de la langue, etc. Il y a là un jeu complexe entre le conscient - à savoir le sens, les idées à exprimer - et les actes moteurs de la parole qui ont basculé dans le réflexe depuis longtemps et n'obéissent qu'à l'effet que l'on veut produire - notionnellement et émotionnellement - et pour lesquels l'interprétation peut agir comme un verre grossissant qui fait apparaître des détails échappant à l'œil nu.» 
Dans un tribunal, tout particulièrement, chaque discours est imprégné des sentiments qui animent le locuteur: l'intonation pour les entendants, l'expression faciale pour les sourds. L'expression faciale est à la langue des signes ce que la prosodie est aux langues vocales.

La voix trahit la peur, la honte, la colère, l'indignation, les regrets, le désespoir et tous les sentiments qui nous animent dans des situations exceptionnelles. De par sa neutralité, l'interprète doit-il adopter une voix neutre? C'est sur ce point qu'il existe une grande confusion entre avoir une voix neutre et rester neutre par rapport au contenu d'un message. Adopter une voix neutre par crainte d'être taxé de partialité est une erreur interprétative. C'est déjà, à mon avis, prendre position et donner l'image de l'indifférence du prévenu face à la situation évoquée. C'est trahir son vouloir-dire, car la reformulation ne produira pas sur le destinataire les effets qu'aurait pu produire l'original. Les mêmes mots dits avec une intonation différente ne traduiront pas le même vouloir-dire. Ce n'est pas la qualité du timbre de voix qui importe, mais la justesse et la sincérité du ton par rapport à l'émotion contenue dans le message.

L'auteur est responsable de son énoncé, tant par le choix des mots que par l'intonation. Vouloir la gommer, c'est manquer d'objectivité. Il ne faut pas tomber dans l'excès inverse et exagérer le caractère prosodique de l'énoncé. Ce serait aller rechercher les intentions, les arrière-pensées du locuteur; l'interprète outrepasserait son rôle; il doit s'en tenir uniquement au message, sans ajout ni omission.

Le rythme comme effet prosodique peut aussi apporter une valeur sémantique à l'énoncé linguistique. Un prévenu inculpé pour outrage public à la pudeur retrace les faits sans la moindre interruption, avec un débit accéléré. Le rythme trahissait peut-être la gêne, la honte de l'acte commis ou la crainte que quelqu'un de connu puisse prendre connaissance de ce qu'il disait.

La recherche d'une explication est du domaine de l'exégèse et n'intervient pas dans mon interprétation; mais l'adoption du rythme m'incombe, car celui-ci produira les effets du message original sur le destinataire, c'est-à-dire le tribunal. J'ai interprété en simultané en m'efforçant de suivre le débit de «parole». Si mes mots se bousculaient presque, mon propre débit corroborait la rapidité des gestes exécutés.

\section{LES TECHNIQUES INTERPRÉTATIVES}

Le choix du mode d'interprétation varie en fonction des différentes phases d'audience — interrogatoire, exposés des faits, réquisitoire, plaidoirie. Lors de l'interrogatoire du prévenu sur son identité, les questions fermées appellent des réponses brèves. Du fait de la brièveté des échanges, la méthode d'interprétation préconisée est la consécutive. Mais dès que le président d'audience demande au prévenu d'exposer les faits, la réponse nécessite un développement. La consécutive n'est plus possible. Entendre un discours sans pouvoir le comprendre peut impatienter et gêner le tribunal. De plus, laisser un temps mort crée un malaise et allonge les débats. La consécutive est déconseillée; elle nuit au rythme du discours et peut perturber le prévenu dans le déroulement de ses idées. De plus, elle pourrait, sous certains aspects, rappeler les procédés utilisés par ceux qui faisaient office d'interprètes il y a quelques décennies. Une pseudo-consécutive masquạit soit un résumé soit au contraire une exégèse des propos du locuteur. Après avoir été victimes d'une falsification de leur "parole», les sourds sont devenus très vigilants sur les compćtences professionnelles des interprètes pour avoir la garantie de l'authenticité de leur discours.

Personnellement, j'opte le plus souvent pour la simultanée. Par rapport à nos confrères interprètes en langues vocales, nous avons l'avantage de ne pas créer de superposition de voix; la simultanée en est d'autant plus facilitée. Les raisons de ce choix visent à éviter un décalage entre l'énoncé original et la reformulation. Ainsi, le prévenu sourd s'exprime 
par ses signes (dans le sens linguistique du terme); sur cet énoncé signé se greffent l'expression faciale, l'amplitude du geste, un certain type de comportement qui sont des apports sur le plan linguistique et psychologique. Associés dans un délai bref à la reformulation oralisée du message, le tribunal perçoit celui-ci dans sa globalité comme s'il érnanait directement du locuteur. L'illustration en est donnée par l'affaire évoquée au paragraphe 3.4 .

Dans le sens tribunal $\rightarrow$ prévenu sourd, la prise en compte de l'interprétation en simultanée n'est pas perçue de la même façon en raison d'un problème sensoriel évident: la personne sourde ne peut pas regarder en même temps son locuteur et l'interprète alors qu'en sens inverse, toute personne entendante peut regarder un sujet et percevoir en même temps un discours émanant d'une autre personne. Le déficit auditif tronque le processus classique de transmission du discours, d'où les adaptations obligées par rapport au receveur du message.

Quelquefois, le tribunal peut nous demander de procéder à une traduction à vue d'un texte: procès-verbal, lettre ou rapport médical. Selon la nature du texte et sa longueur, la non-connaissance préalable de celui-ci peut être une gêne à une restitution fidèle. Dans une attitude bienveillante, pour faciliter notre tâche, le tribunal peut nous inviter à faire un résumé. Le problème n'est pas résolu pour autant. Est-ce à nous qu'incombe la responsabilité de faire le résumé ? Est-ce la solution à l'égard de tout justiciable?

$\mathrm{Au}$ cours d'un procès, un justiciable sourd quasiment illettré et presque asocial devait, selon la loi, prendre connaissance par mon truchement des conclusions du rapport du médecin psychiatre. Ce rapport médical, rédigé dans un technolecte, ne pouvait être accessible qu'à des personnes de niveau socio-culturel équivalent et pour qui les concepts étaient évocateurs. Le président, conscient que le contenu du texte n'était pas à la portée du prévenu, me demande de lui signifier qu'il est jugé responsable de ses actes, donc soumis à une sanction pénale. Ce simple énoncé était déjà très complexe pour cet homme. En voulant rendre le message encore plus simple, le président me propose cette phrase lapidaire : «Dites qu'il n'est pas fou.»

Si en mathématiques: «(-) par $(-)=(+) »$, cette équivalence est difficilement transposable sur le plan linguistique. Un énoncé produit à la forme affirmative n'a pas la même valeur sémantique que son contraire à la forme négative. Les textes juridiques jonglent subtilement avec des phrases insérant la double négation, dont voici une des plus connues: «Nul n'est censé ignorer la loi.» Même si cette phrase semble proche de: «Tout le monde doit connaître la loi», ces deux unités de sens ne recouvrent pas la même surface sémantique et surtout ne produisent pas les mêmes effets sur le destinataire du message.

Ainsi donc: «être jugé responsable de ses actes» n'est pas l'équivalent de: «être jugé ne pas être fou». De plus, le message très court semble mettre en exergue le mot «fou». Ce terme banalisé dans le langage courant pour les personnes entendantes, comme pour les personnes sourdes, revêt une connotation très forte, étant donné les circonstances, surtout sur un esprit assez primaire. Une réaction violente était à craindre de la part du prévenu de nature impulsive. D'où mon choix de procéder à une démarche didactique pour faire passer les concepts de: connaissance/acte responsable - sans pour autant utiliser les signifiants correspondants puisqu'ils étaient inconnus.

Dans le cas de ce prévenu, avec l'approbation du magistrat, j'ai tenté d'extrapoler sa situation personnelle en évoquant différents délits. J'associais à chaque fois le fait que l'auteur dudit délit comparaissait devant le tribunal pour y être jugé. La relation entre délit et sanction semblait être admise par le prévenu par ses mouvements de tête approbateurs. J'ai abandonné la phase explicative pour recentrer mes propos sur les conclusions du rapport du médecin psychiatre par une phrase que je cite de mémoire. «Le médecin a écrit que vous savez qu'il est interdit de voler de l'argent, une voiture ou tout autre chose, 
de taper et blesser qui que ce soit ou d'entrer par effraction chez quelqu'un, etc., sous peine d'être jugé par un tribunal et puni suivant la loi. Ainsi vous, avec des copains, vous êtes entré par effraction dans une maison pour voler, vous serez jugé selon la loi.»

Certains pourront penser que nous nous éloignons de l'interprétation stricte telle que nous devons la concevoir; mais, face à une situation aussi désarmante, nous sommes tenus d'imaginer des stratégies inédites pour faire passer le message.

Des procédés didactiques sont fréquents pour pallier des déclivités culturelles ou des carences lexicales dont pâtit la langue des signes, surtout en ce qui concerne le technolecte juridique.

\section{LES PROBLÈMES LIÉS À LA MÉCONNAISSANCE DE LA SURDITÉ 5.1. LES DÉNOMINATIONS}

Sans faire un exposé sur la surdité, quelques explications permettront d'éclairer différentes stratégies d'interprétation adaptées à la spécificité de la situation.

Les personnes sourdes ont été désignées par différentes dénominations, engendrées par la philosophie d'une société à une époque donnée à l'égard des personnes déficientes auditives: muet, sourd-muet, malentendant. Les personnes non averties ne savent pas toujours si ces différentes dénominations renvoient à des caractéristiques médicales ou s'il existe une synonymie entre elles pour distinguer une même catégorie de personnes atteintes d'un même handicap sensoriel, ce qui a créé des confusions et des situations ambiguës.

Le terme sourd-muet, ancien, désuet, affublé d'une connotation très négative, entretient l'image d'un individu affligé d'une infirmité aggravée de faibles capacités intellectuelles. Si, sur le plan médical, il existe des sujets atteints de surdité et de mutité, ils représentent des cas isolés, au moins sur notre territoire.

On imagine facilement la surprise, voire la stupéfaction du tribunal, quand un justiciable présenté comme sourd-muet s'exprime dans un français correct avec de surcroît une élocution compréhensible. L'ignorance entraîne l'incompréhension, et la frontière avec la suspicion est mince. L'interprète auprès des tribunaux a le titre d'expert, c'est-à-dire qu'il peut de par ses connaissances extra-linguistiques, et en l'occurrence ses connaissances sur la surdité et des problèmes inhérents, apporter aux magistrats des éclairages absolument indispensables pour une meilleure compréhension du contexte ou pour mieux saisir la pertinence du message.

Le terme sourd est plus couvrant, utilisé pour désigner des personnes atteintes de surdité profonde survenue à la naissance ou au cours de la petite enfance. Le terme malentendant est attribué à ceux qui ont une déficience auditive moyenne ou sévère et vise particulièrement les devenus sourds à l'âge adulte qui refusent l'appellation de sourd. Ils sont totalement distincts du groupe précédent et notre concours vis-à-vis d'eux est d'une autre nature.

La confusion des dénominations se porte également sur celles des modes de communication: interprète en langage mimique, en langage gestuel, en sourd-muet, ce qui ne manque pas d'humour, et, de façon plus académique, interprète en langue des signes française. Cette palette de vocables tend à démontrer que la surdité est un handicap mal connu car invisible; il gêne et déroute l'entendant en tant que locuteur potentiel. La gêne est plus marquée qu'entre deux locuteurs entendants de langue différente, même si le résultat est identique.

\subsection{LA COMMUNAUTÉ SOURDE}

Comme toute communauté linguistique, elle se distingue par sa langue, son histoire, ses traditions, sa culture. Mais elle se singularise par l'absence d'un territoire géographique délimité, par sa langue qui se transmet par ses pairs et non par ses pères, puisque 
la surdité n'est pas héréditaire et par ce dénominateur commun qu'est la déficience auditive. Cette minorité linguistique a connu son développement autour des établissements scolaires spécialisés en constituant ainsi des microcosmes qui se perpétuent au sein de vies associatives. Des liens privilégiés se sont tissés entre les membres de cette communauté.

De ce fait, les interprètes, soit des personnes entendantes issues de cette communauté possédant la langue des signes comme langue maternelle, soit des personnes extérieures à celle-ci ayant la maîtrise de cette langue, deviennent des personnages phares, de par leur place et de par leur fonction de médiateur, et ne peuvent passer inaperçues. Cette notoriété inévitable peut occasionner de sérieux inconvénients. Les facteurs relationnels peuvent gêner la sérénité des échanges ou engendrer des jugements subjectifs sur les compétences de l'interprète.

\subsection{LES DIFFÉRENTS MODES DE COMMUNICATION}

Du fait des différents degrés de surdité, des apports éducatifs plus ou moins précoces, des différents milieux socio-culturels, les modes de communication varient : allant de la langue des signes, langue clairement codifiée, telle qu'elle est pratiquée en conférence, à des pidgins plus ou moins élaborés jusqu'au mime le plus rudimentaire.

Certains sourds, habitués à côtoyer des personnes entendantes, associent leur communication signée à une labialisation plus ou moins importante des mots de la langue française. Dans tous les cas de figures, il importe pour les interprètes d'être polyvalents, c'est-à-dire d'avoir la faculté d'adaptation aux différentes situations de communication.

Lorsque le justiciable sourd est invité à s'exprimer, il peut le faire par signes tout en ponctuant son énoncé de mots oralisés appartenant à la langue française. Mais la connaissance du français est le plus souvent artificielle et mnémonique. Cette situation linguistique est comparable à celle des justiciables francophones originaires d'Afrique que présente Christiane Driesen (1985) dans sa thèse.

La superposition des deux modes de communication, oral et gestuel, ne gêne pas l'interprète; la communication est «multicanale», le sens du message est unique. La gêne vient du fait de l'oralisation de certains mots perçus par les magistrats qui leur attribuent une valeur sémantique autre que celle voulue par le locuteur sourd, ce qui peut faire douter les magistrats de la fiabilité de notre interprétation.

\section{PROBLÈMES DUS À LA NATURE DE LA LANGUE DES SIGNES \\ 6.1. LE VIDE LEXICAL}

De par sa nature, la langue des signes est une langue de proximité et de par son histoire, elle s'est longtemps maintenue dans un statut de langue de convivialité; de ce fait, son lexique s'est peu étoffé et présente des carences lexicales, dans certains domaines spécifiques. Ainsi, face au technolecte juridique en référence au Code pénal et au sociolecte des magistrats, l'interprète doit recourir à des procédés didactiques.

Afin de comprendre ce langage de spécificité, l'interprète doit avoir obligatoirement des connaissances juridiques. Sans chercher à égaler le savoir d'un magistrat, il doit être familiarisé avec les termes juridiques et les notions auxquelles ils renvoient.

\subsection{LA FAUSSE TRANSPARENCE DES SIGNES}

Les personnes non averties font souvent l'amalgame entre les gestes de la communication non verbale et les signes de la langue des signes. Cette confusion est tout à fait explicite.

Le geste est le propre de l'homme; il nous est familier par sa présence dans la communication à des degrés divers selon les locuteurs. Comme élément coverbal dans le discours, il accompagne la parole en illustrant une description, soulignant une démonstration, rythmant une énonciation, accentuant des prises de position. Il peut se suffire également à 
lui-même comme quasi-linguistique; son utilisation reste toutefois occasionnelle de par la brièveté de son information et des situations particulières des locuteurs: milieu bruyant, éloignement ou langage professionnel codé.

Tout en reconnaissant que la langue des signes a puisé ses racines dans la gestualité humaine, elle a depuis longtemps transcendé ce stade. De par leur intelligence, les sourds ont fait opérer à celle-ci un saut qualitatif lui permettant de passer de l'état de langage à celui de langue.

La familiarité des gestes dans certaines situations conversationnelles donne l'illusion à certaines personnes de pouvoir comprendre les signes de la langue des signes. C'est ce que $\mathrm{j}$ 'appelle la fausse transparence.

Deux types d'erreurs sont fréquents:

1. L'illusion de la compréhension est provoquée par une fausse similitude avec les signes coverbaux; un seul des composants du signe change: configuration de la main, point de contact avec le corps, mouvement dans l'espace... et la signification diffère.

2. Si toutefois la similitude est réelle, la signification est le plus souvent différente de celle attribuée par les personnes entendantes et, dans un discours, le sens de l'énoncé est tout autre que celui du sens présupposé. Cette fausse facilité rejoint les propos tenus par D. Seleskovitch concernant la traduction des langues proches. La similitude des mots crée un phénomène «d'hypnose». «Plus le mot est proche, plus les langues sont proches, plus la difficulté d'une bonne traduction est grande» (1986: 134). Fortuna, sinistra en italien ne signifient pas respectivement: fortune et sinistre en français mais chance et gauche.

L'interprète ou le traducteur de métier sait éviter ces pièges linguistiques, mais comment empêcher l'initiative interprétative chez des non-professionnels, surtout concernant la langue des signes? Ils ont vu, ils ont cru discerner, donc ils ont compris.

Il faut non seulement à l'interprète de bonnes compétences professionnelles mais également «des intuitions linguistiques» pour deviner ce qui, dans un discours antérieur, aurait pu faire l'objet d'une fausse interprétation de la part de personnes entendantes ignorant la langue des signes mais pensant la comprendre par intuition.

Lors d'une instruction, le prévenu sourd devait s'expliquer sur les raisons qui l'avaient poussé à faire des menaces de mort à l'encontre d'une jeune femme entendante dont il était amoureux. Celui-ci ne semblait pas comprendre les motifs d'accusation. Il voulait bien admettre qu'ayant été éconduit il s'était emporté, mais niait le fait d'avoir proféré des menaces de mort. Face aux négations du prévenu, le juge d'instruction procède à une confrontation. La jeune femme ne connaissait pas la langue des signes et pourtant elle était sûre d'avoir compris que le prévenu voulait lui trancher la gorge. Grave accusation que nie le prévenu. Ses problèmes psychologiques et son comportement ne plaidaient pas en sa faveur; le doute semble subsister chez le juge. Comme expert, je me permets d'intervenir auprès du juge en évoquant les erreurs d'interprétations des signes si l'on ne connaît pas la langue. La jeune femme est invitée à refaire les gestes de menace proférés à son encontre. Pour toute personne connaissant les deux langues, français et langue des signes, la méprise était évidente. Le signe restitué signifiant «assez» et celui signifiant «trancher la gorge» étaient très proches. Le contexte de la querelle favorisait la confusion entre les deux gestes, entraînant des interprétations erronées.

L'interprète peut, comme expert, apporter des informations extérieures à l'affaire pour contribuer à une meilleure compréhension d'une situation, tout en restant à sa place d'interprète. En référence à l'affaire citée, l'expérience professionnelle et la connaissance des deux milieux linguistiques sont des atouts majeurs pour interpréter juste et aussi pour cibler les informations qui peuvent être utiles aux magistrats. 


\subsection{LA DÉCLIVITÉ CULTURELLE}

Les obstacles linguistiques vont de pair avec la déclivité culturelle. Ce n'est plus la langue qui est à défaut, c'est l'individu qui est très limité dans ses potentialités linguistiques. «Lorsqu'un locuteur dispose d'un code restreint, il y a déficit de la communication» (Ladmiral 1994: 143). D'où des conséquences graves pour un justiciable, malgré certaines circonstances atténuantes. «Nul n'est censé ignorer la loi.»

Les notions d'interdit, de droit, d'obligation, de bien, de mal sont liées à nos lois, à nos valeurs morales, à notre éducation. La langue a été le vecteur qui nous a permis d'acquérir ces concepts. La non-mâ̂trise du français oral et écrit, liée à des carences éducatives, entraîne des critères de valeur différents des nôtres. D'où l'impossibilité pour certains prévenus de comprendre le caractère délictueux du recel, de la complicité, du droit légal de perquisition de la police à votre domicile si elle en a été mandatée.

La déficience communicationnelle se traduit pour certains sourds par des énoncés brefs et imprécis où la langue des signes et la mimique se trouvent étroitement mêlées. Le message n'est pas toujours pertinent. Si l'interprète a connaissance du contexte situationnel, il retrouve le sens exact du message en palliant les carences linguistiques du locuteur. Dans le cas contraire, il ne peut que faire des hypothèses de sens mais ne peut en rester à ce stade. Aussi doit-il prévenir le tribunal des difficultés de communication et provoquer des questions par l'intermédiaire du président qui reste maître du dialogue. Des réponses à des questions très précises peuvent lever les ambiguïtés du message original.

Je voudrais souligner les difficultés toutes particulières de la reformulation dans les situations délicates concernant les affaires d'attentat à la pudeur, viol et inceste lorsque le prévenu ou la victime n'a accès qu'au langage mimique.

Chaque langue possède un lexique suffisamment explicite pour parler des faits; plus les actes sont répréhensibles, plus les termes semblent éloignés du langage courant en voulant jeter un voile pudique sur ce qu'ils évoquent.

Pour un même concept, les mots dits n'ont pas le même impact que les signes vus. Cela tient à l'aspect formel des signifiants. Par leur caractéristique gestuelle et leur apparente iconicité, les signifiants de la langue des signes prennent une connotation particulière pour des entendants dont les usagers de cette langue des signes n'ont pas toujours conscience. Ce qui est naturel pour les uns peut être choquant pour les autres.

L'impression de gêne est accentuée si le code linguistique est remplacé par un pidgin proche du mime. Les interprètes débutants sont souvent très mal à l'aise surtout en audience quand tous les yeux sont braqués sur eux comme s'ils étaient le clou du spectacle.

Les présidents sont en général conscients de cette gêne et peuvent être amenés à prendre des dispositions telles que le huis clos pour faciliter l'interprétation et assurer la sérénité des débats.

\section{L'INTERPRÈTE : SON RÔLE - SES COMPÉTENCES}

\subsection{SON RÔLE}

L'interprète a un rôle de médiateur, d'agent de la communication. Il doit placer la personne étrangère ou sourde dans les mêmes conditions que l'autochtone ou l'entendant. Son rôle se définit par ses obligations et par les limites de sa fonction.

Par sa présence et son concours, l'interprète permet aux interlocuteurs de franchir la barrière des langues et d'établir entre eux un contact dont il a la responsabilité. Même s'il ne participe pas aux échanges en tant qu'auteur des messages, il n'en est pas moins actif. Grâce à ses compétences, il permet le bon fonctionnement de la communication. Dans son ouvrage L'interprète dans les conférences internationales (1968: 182), D. Seleskovitch nous fait part de sa conception du rôle de l'interprète de conférence. Cette conception rejoint, à mon avis, celle que nous devons avoir de notre propre rôle au pénal. 
L'interprète conscient de son apport personnel au bon déroulement de la conférence sait tirer une ligne de démarcation très nette entre l'intervention qui correspond à l'exécution de sa tâche (faire se comprendre les interlocuteurs), et qui fait de lui un participant au «trilogue» et celle qui le ferait abusivement intervenir dans le «dialogue»s'il teintait de ses propres convictions les messages qu'il transmet.

L'interprète doit transmettre fidèlement le message dans son intégralité avec la plus grande impartialité. Être fidèle en interprétation, c'est être fidèle au sens du message, au vouloir-dire de l'auteur, à l'impression que celui-ci veut produire sur son destinataire.

Ce principe fondamental est souligné par la justice puisqu'il figure dans la formule de prestation des traducteurs experts; «Vous jurez de traduire fidèlement tout ce qui vous sera rapporté...» art. 20 du décret $n^{\circ} 74-1184$ du 31 décembre 1974.

Tout manquement à cette exigence peut entraîner des conséquences pour le prévenu ou pour la partie civile. Par le choix des mots, leurs nuances, leurs connotations, par l'intonation d'une phrase, l'interprète peut influer sur le jugement de la personnalité du justiciable et sur les décisions du tribunal.

Mais la retransmission fidèle du sens d'un énoncé ne doit pas conduire l'interprète à rechercher des explications sur les intentions de l'auteur. Il transmet clairement le message exprimé sans se préoccuper des motivations qui poussent celui-ci à parler ainsi. Les intentions, les arrière-pensées du prévenu ne nous appartiennent pas, même si on les devine. Elles ne font pas partie du discours. «Le sens d'une phrase c'est ce qu'un auteur veut délibérément exprimer, ce n'est pas la raison pour laquelle il parle, les causes ou les conséquences de ce qu'il dit. Le sens ne se confond pas avec les mobiles ou des intentions. Le traducteur qui se ferait exégète, l'interprète qui se ferait herméneute transgresserait les limites de ses fonctions» (Seleskovitch 1986: 269).

Dans les interprétations en milieu judiciaire, le dérapage vers l'exégèse est tentant du fait de la pression psychologique que subit, même indirectement, l'interprète. Celui-ci doit bien cerner l'objectivité du sens et s'y conformer dans sa reformulation.

Pour ne pas être taxé de partialité, l'interprète doit respecter les limites de ses fonctions et observer une totale neutralité; elle se manifeste vis-à-vis du prévenu, vis-à-vis du tribunal, vis-à-vis de l'affaire jugée. Il n'a pas à faire part de ses sentiments personnels, ni en approuvant, ni en désapprouvant ce qui est dit. Il n'est ni moralisateur ni avocat.

\subsection{SES COMPÉTENCES}

Comme l'interprétation n'est pas une simple opération linguistique, elle est soustendue par des connaissances extra-linguistiques qui regroupent à la fois un certain savoir encyclopédique, les connaissances contextuelles, et aussi des connaissances juridiques. Sans vouloir rivaliser avec un juriste ou un magistrat, des connaissances sur les différentes instances judiciaires, sur le technolecte juridique et les concepts qui y sont associés s'imposent.

Ce qui est absolument indispensable, ce sont les connaissances concernant la surdité : les aspects médicaux, éducatifs, psychologiques, socio-culturels. Ces connaissances sont les compléments indispensables pour assurer une bonne interprétation et pour justifier le rôle d'expert que l'interprète est amené à prendre dans des circonstances précises.

Toutes ces connaissances s'associent impérativement à une formation de haut niveau. C'est par l'apprentissage des méthodes interprétatives et leur application que l'interprète prouvera sa compétence et son efficacité.

Comme nous l'évoquions au début de cet article, l'histoire de la communauté sourde et l'histoire de sa langue vont de pair. Le discrédit jeté sur cette langue a annihilé toute évolution linguistique et a paralysé ceux qui auraient pu l'enrichir. 
Ainsi, ceux qui faisaient office d'interprètes connaissaient la langue, certes, mais sans avoir jamais reçu de formation. Grâce à leur bonne volonté, ils ont permis à la retransmission d'informations et au dialogue de s'instaurer entre locuteurs. Mais la bonne volonté n'est pas un label de qualification et, aujourd'hui, la communauté sourde est devenue à juste titre exigeante sur la qualité de l'interprétation.

L'ESIT permet aujourd'hui de répondre à l'attente des personnes sourdes en ouvrant ses portes aux interprètes pour sourds. Depuis 1993, ceux-ci peuvent préparer une M.S.T. - Maîtrise de Science et Technique de l'interprétation - en se confrontant aux mêmes exigences de formation que les interprètes des langues vocales.

Cette mise en œuvre est la meilleure preuve de la reconnaissance du droit des personnes sourdes à la retransmission authentique de tout message, ce qui par là même assure la revalorisation de la langue des signes.

\section{CONCLUSION}

Au cours de cette présentation, partant de mon expérience personnelle et de mes convictions, j'ai voulu confirmer deux idées essentielles.

L'interprétation pour sourds ne déroge pas aux principes fondamentaux de l'interprétation: la théorie du sens et le processus triangulaire de l'activité indépendamment des langues utilisées. «La compréhension n'est pas autre chose que la réduction de l'information au sens, exprimable ensuite dans n'importe quel code de communication» (Seleskovitch 1968:83).

L'interprétation pour sourds recouvre les mêmes spécificités que toute autre interprétation au pénal : contexte particulier du prétoire, obstacles socio-culturels. Elles sont accentuées par la confrontation d'une langue à caractère vocal à une langue à caractère gestuel et par la complexité des problèmes que crée la surdité.

Quelles que soient les particularités de la situation, que le justiciable soit sourd ou étranger, l'interprète au pénal doit avoir un même but: placer celui-ci dans les mêmes conditions que le justiciable entendant ou autochtone.

Par égard pour la mission qui lui est confiée, l'interprète requis doit être un professionnel digne de ce nom, afin d'assurer une interprétation de qualité dans un esprit d'honnêteté envers le prévenu et de respect envers la justice.

\section{RÉFÉRENCES}

DRIESEN, Ch. (1985) : «L'interprétation au pénal en R.F.A. - L'obstacle linguistique», Thèse en traductologie, disponible à l'ESIT, Université Paris III, p. 13.

LADMIRAL, J.-R. (1994): Traduire : théorèmes pour la traduction, nouvelle édition, Paris, Gallimard, p. 143. LEDERER, M. (1973) : «La traduction : transcoder ou réexprimer», ELA, $\mathrm{n}^{\circ}$ 12, Paris, Didier, p. 21.

SELESKOVITCH, D. (1968): L'interprète dans les conférences internationales, Paris, Lettres modernes Minard, pp. 182 et 183.

SELESKOVITCH, D. (1976) : «De l'expérience aux concepts», ELA, n² 24, Paris, Didier, pp. 66 et 73.

SELESKOVITCH, D. (1986): Interpréter pour traduire : Exégèse et linguistique, Paris, Publications de la Sorbonne, Didier Érudition, p. 269.

SELESKOVITCH, D. (1986): Interpréter pour traduire : Les niveaux de traduction, Paris, Publications de la Sorbonne, Didier Érudition, p. 134. 